7?

columns after a normal electrocardiogram at the request of the family, when the psychosis re-emerged. A literature search also suggested this was a suitable drug following NMS. Sodium valproate was added for treatment of the epilepsy but has also been a very effective mood stabiliser

Interestingly, when the patient emerged from her catatonic state, she used the hemiplegic arm without any difficulties for a few days before it returned to a spastic premorbid position Finally, the family are extremely pleased with the patient's progress, believing her mental state to be the best they have ever seen it and this is echoed by all staff who have known this woman.

Jennifer Dolman Specialist Registrar, Rosemary Baker Consultant Psychiatrist, Learning Disability Service, The Hollies, Parklands Hospital, Aldermaston Road, Basingstoke, Hampshire RG24 9RH

\section{Clinical capacity assessment}

Sir: Dr Raymont (Psychiatric Bulletin, February 2002, 26, 201-204) is right to draw to our attention the complexities involved in the legal basis of our ministrations to the patient who may lack the capacity to give informed consent. We especially welcome discussion of the issue of belief and insight in this philosophical, legal and ethical morass, although we would have liked to see elaboration of terms like 'full insight' and 'greater level of capacity'. However, a particular suggestion made us wince.

Dr Raymont answers her own question, 'So how can we proceed currently with any physical treatment of those who lack capacity?' with 'Certainly a full psychiatric assessment should be made initially' apparently before life-saving treatment. This presumably applies to many with major stroke or an acute cardiac event, a large number of the 30-60\% admitted to medical wards with dementia or delirium (Ramsay et al, 1991; Treloar \& Macdonald, 1997), a high proportion of all those in nursing homes (Macdonald et al, 2002) and every single unconscious patient. If by 'psychiatric assessment' she includes presenting complaint, history of presenting complaint, collateral history, and so on by mental health professionals, we wonder where all these professionals will come from?

Apart from this practical problem, we object on principle to the growing tendency for physicians and surgeons to involve psychiatrists in judgements about capacity to consent. Under current UK law (as opposed to some of the US jurisdictions in which the MacArthur Competence Assessment Tool for Treatment (MacCAT-T) (Appelbaum \& Grisso, 1998) was developed) it is not necessary to diagnose the cause of any impaired capacity in order to make the judgement that it is impaired. All professionals must surely be able to make such judgements in relation to each decision, great or small, confronting their patient if they are not to be constantly exposed to accusations of battery on the one hand or neglect of duty of care on the other.

Each trust must ensure that its doctors are competent to assess capacity and have policies in place for treatment when capacity is lacking. It is the responsibility of every treating physician to gain the informed consent for the treatment they are delivering and to take appropriate measures if they do not believe the person to have that capacity. These include timely interventions to save life and, when more leisurely interventions are allowed, involving the relatives, consulting colleagues (almost never a psychiatrist) and other measures, in accord with the Bolam standard (Bolam v. Friern, 1957).

APPELBAUM, P. S. \& GRISSO,T. (1998) MacArthur CompetenceAssessment Tool for Treatment. Sarasoto, FL: Professional Resource Exchange.

MACDONALD, A. J. D., CARPENTER, G. I., BOX, O., et al (2002) Dementia and use of psychotropic medication in non- 'Elderly Mentally Infirm' nursing homes in South East England. Age \& Ageing, 31, 58-64.

RAMSAY, R.,WRIGHT, P., KATZ, A et al (1991) The detection of psychiatric morbidity and its effect on outcome in acute elderly medical admissions. International Journal of Geriatric Psychiatry, 81 861-866.

TRELOAR, A. J. \& MACDONALD, A. I. D. (1997) Outcome of delirium diagnosed by DSM - III- $R$ ICD-10 and CAMDEX, and derivation of the reversible cognitive dysfunction scale among acute geriatric inpatients. International Journal of Geriatric Psychiatry, 12, 609-613.

Bolam v. Friern. Hospital Management Committee [1957] 2AllER 118, 1 W:R 582

Chris Ball Consultant, Old Age Psychiatry, Alastair Macdonald Professor of Old Age Psychiatry, King's College London, Ladywell House, 330 Lewisham High Street, London SE13 6JZ

\section{Off-label prescribing}

Sir: We enjoyed reading the article by Lawrence et al (Psychiatric Bulletin, June 2002, 26, 230-232). The findings complement some work that we have done in this area (Lowe-Ponsford, Psychiatric Bulletin, 2000, 24, 415-417). Our postal questionnaire found that $65 \%$ of psychiatrists who replied had prescribed 'off-label' within the preceding month.

Medico-legal advice that we obtained (from the Medical Protection Society) was that, not only does Bolam (1957) need to be taken into account, but also the case of Bolitho (1997). This judgement means that the treatment has to withstand logical analysis as well as be accepted by a body of opinion. These considerations need to be taken into account in prescribing, alongside the capacity of the patient.

Many psychiatrists are worried about off-label prescribing and our study showed that $4 \%$ of respondents had received complaints about this matter. In our paper, we suggested some guidelines that may avoid many future medico-legal problems for clinicians if they should prescribe off-label.

The College's Psychopharmacology Special Interest Group has discussed this matter and is setting up a small working group (chaired by D. B.) to review the practice of off-label prescribing. We would be delighted to receive the thoughts of colleagues on this subject.

Bolam v. Friern. Hospital Management Committee [1957] 2AllER 118, 1 W:R 582

Bolitho v. City and Hackney Health Authority [1997] 3 WLR1151.

Francesca Lowe-Ponsford Locum Consultan Psychiatrist, Box 317, Adrian House, Fulbourn Hospital, Cambridge CB1 5EF, David Baldwin Senior Lecturer in Psychiatry, University Department of Mental Health, Royal South Hants Hospital, BrintonsTerrace, Southampton SO14 OYG

\section{Community psychiatry in Nigeria}

Sir: Accredited accommodation: an alternative to in-patient care in rural north Powys (Readhead et al, Psychiatric Bulletin, July 2002, 26, 264-265).

Although separated by historical period, civilisation and culture, it is interesting to compare this scheme to a similar one in a developing country. The 'Aro village' in Nigeria is set in a semi-rural culture, and operated by the Department of Psychiatry, University of Ibadan. It was pioneered by Professor Adeoye Lambo, an eminent psychiatrist who later became the Deputy-Director of The World Health Organization. This initiative was the very first attempt in community psychiatry in Nigeria (Boroffka \& Olatawura, 1976).

As in the case of the north Powys project, Aro village was adapted from the already existing infrastructure of a village community. It offered a social model of care and a rich rehabilitation resource.

Community confidence in the scheme was achieved through liaison between the psychiatrists and the community leaders, a delicate balance between traditionalists and Western psychiatry, a relationship based on trust and the prospect of mutual benefits from the project. In return, the Aro village witnessed infrastructural developments and on-site health clinics. Among other research interests, the project was the subject of an international research collaboration by Leighton et al, 1963 
In a self-funded health economy, patients and their families paid for lodging in the village. The period of stay was longer, at times it ran into years, and some patients relocated to live permanently in the village.

Finally, the benefits were not couched in terms of econometrics, but in the confidence of the rural community to embrace the, then, alien Western psychiatry.

BOROFFKA, A. \& OLATAWURA, M. O. (1976) Community psychiatry in Nigeria: the current status. International Journal of Social Psychiatry, 23, 1154-1158.

LEIGHTON, A. H., LAMBO, T. A., HUGHES, C., et al (1963) Psychiatric Disorder among the Yorubas: A Report. NewYork: Cornell University Press.

Ade Adewunmi Consultant Psychiatrist Calderstones Hospital, Mitton Road, Whalley, Clitheroe, Lancashire BB7 9PE

\section{Military psychiatry}

Sir: Military psychiatry is, as Greenberg et al (Psychiatric Bulletin, June 2002, 26, 227-229) suggest, a unique national resource. I agree (Psychiatric Bulletin, July 1997, 21, 418-421) that it should focus on those areas in which it can justly claim expertise.

Greenberg et al state that military psychiatry is essentially occupational and they mention its 'ethical and moral' challenges. As a serving Officer, I was certainly aware of ethical tensions. On the whole, service personnel came to see me as a psychiatrist, not as an occupational physician. The occupational role, however, meant that under certain circumstances I had to act, not primarily in the best interests of the patient, but to safeguard the military. This possibility was not made explicit. Yet, military patients had few options but to be seen by military psychiatrists.

In civilian life, we can make more autonomous decisions concerning whom we see. The role of the occupational physician is explicit. Doctors only normally act against the wishes of their patients when there are substantial risks associated with not doing so. This is not always the case in the military, because the rules relating to mental illness are attuned to the needs of the organisation, not to personal needs. For understandable (but none the less stigmatising) reasons, the military environment is intolerant of what it perceives as mental illness. So, there can be an ethical tension between the occupational role and the therapeutic inclination. This ethical dilemma is another unique feature of military psychiatry and one that it would be interesting to see addressed.
Julian C. Hughes Gibside Unit, Centre for the Health of the Elderly, Newcastle General Hospital Westgate Road, Newcastle-upon-Tyne NE4 6BE

\section{Overseas candidates for the MRCPsych examination}

Sir: I read with great interest the article by Tyrer et al (Psychiatric Bulletin, July 2002, 26, 257-263) on the MRCPsych examination.

The major findings of their study were: (1) doctors from the UK/Ireland fare much better than their counterparts from the rest of the world; and (2) the older the candidate, the lower the likelihood of success.

I would like to focus on the first point In addition to the putative explanatory factors put forward by the authors to account for this finding, I would like to consider a few more. Compared to UK/ Ireland graduates, overseas doctors are more likely to work in non-teaching hospitals for the whole of their basic specialist training. This will obviously impact on the quality of training they receive and, therefore, their level of preparedness to face the MRCPsych examination. Also, a not insignificant number of overseas trainees are forced by circumstances to take up non-career grade posts for visa purposes: permitfree training (PFT) visas for senior house officers (SHOs) are usually given by the Home Office for a total of 4 years. Understandably, for some, this period is insufficient to pass the examination. The overseas doctor, who sits his subsequent attempts at the MRCPsych examination as a staff grade, will obviously not have the same access to exam-related training as an SHO.

A study that controls for these potential confounding factors (Hospital of training: teaching $v$ non-teaching, and post of the candidate: training $v$ non-career grade) may be able to provide a more accurate picture.

From Table 1, in the Part I examination, $74 \%$ (305/410) of UK/Ireland graduates passed the multiple choice question (MCQ) examination compared with 57\% $(408 / 718)$ of overseas graduates, an absolute difference between the two groups of $17 \%$. Among those successful in the MCQ component, 85\% (206/305) in the UK/Ireland group and 54\% (219/408) in the overseas group were also successful in the clinical component, an absolute difference of $31 \%$. Why does the gulf between the two groups widen from 17$31 \%$ in just a few weeks between the written and clinical examinations? Is the clinical examination unintentionally biased against the overseas trainee?

Another striking statistic in the study is the relative percentage of overseas candidates appearing in the Part I and Part
II examinations. Although in Part I, 64\% $(718 / 1128)$ are foreign graduates, in Part II this drops sharply to just $47 \%$ (357/763) with a corresponding increase in the UK/ Ireland group from 36-53\%. There may be several reasons for this, for example inability to pass Part I, dropping out of psychiatry or moving to non-training posts. Perhaps this is an area for further investigation?

It should not be forgotten that the Professional and Linguistics Assessment Board ( $P L A B$ ) test, which most overseas doctors have to pass to work in the UK, is comprehensive and recognised to be of a high standard.

Finally, the College needs to be appreciated for its transparency in publishing the details relating to the MRCPsych examination. Also, it is encouraging to learn that there is going to be greater standardisation of the examination with the introduction of more reliable sections.

Sundararajan Rajagopal Specialist Registrar, Department of Liaison Psychiatry, Guy's Hospital, York Clinic, Weston Street, London SE1 3RR

\section{Examination performance feedback}

Sir: At present, only candidates who fail the College Membership examination are provided with performance feedback. The feedback is most useful, detailed and helpful in illustrating not only areas of weakness but also positive aspects in the failed performance. The information provided is invaluable to one's success in future attempts.

We believe that it is equally important that successful candidates are given the same opportunity to scrutinise their performance through similarly constructive feedback from the College.

The margin between success and failure may be only slight in a sizeable minority of cases and it is clear that, even after a success, room for improvement remains. With a detailed breakdown of one's performance, there could be at least some pointers for future learning.

We should stress that this suggestion is made with the intention of enhancing one's knowledge of one's abilities, certainly not to provide grounds for appeal nor to provide information for the construction of specialist registrar league tables! There is, naturally, a possibility that such a move may result in the deflation of one's moment of triumph. However, this can be avoided by allowing sufficient time between the euphoria of success and arrival of the detailed feedback for the successful candidates.

T. Glover Specialist Registrar， E. Salib Consultant in Old Age Psychiatry, Hollins Park Hospital, Hollins Park, Warrington 